archives-ouvertes

\title{
Apparent yield stress in rigid fibre suspensions: the role of attractive colloidal interactions
}

\author{
S. Bounoua, E. Lemaire, J. Férec, G. Ausias, A. Zubarev, P. Kuzhir
}

\section{To cite this version:}

S. Bounoua, E. Lemaire, J. Férec, G. Ausias, A. Zubarev, et al.. Apparent yield stress in rigid fibre suspensions: the role of attractive colloidal interactions. Journal of Fluid Mechanics, Cambridge University Press (CUP), 2016, 802, pp.611-633. 10.1017/jfm.2016.475 . hal-01356268

\section{HAL Id: hal-01356268 \\ https: / hal.archives-ouvertes.fr/hal-01356268}

Submitted on 25 Aug 2016

HAL is a multi-disciplinary open access archive for the deposit and dissemination of scientific research documents, whether they are published or not. The documents may come from teaching and research institutions in France or abroad, or from public or private research centers.
L'archive ouverte pluridisciplinaire HAL, est destinée au dépôt et à la diffusion de documents scientifiques de niveau recherche, publiés ou non, émanant des établissements d'enseignement et de recherche français ou étrangers, des laboratoires publics ou privés. 


\title{
Apparent yield stress in rigid fibre suspensions:
}

\section{The role of attractive colloidal interactions}

\author{
S. Bounoua ${ }^{1}$, E. Lemaire ${ }^{1}$, J. Férec ${ }^{2}$, G. Ausias ${ }^{2}$, A. Zubarev $^{3}$, and P. Kuzhir ${ }^{1} \dagger$ \\ ${ }^{1}$ University of Nice-Sophia Antipolis, CNRS UMR7336, Laboratory of Condensed Matter Physics, Parc Valrose, \\ Nice 06100 France \\ ${ }^{2}$ Institut de Recherche Dupuy de Lôme (IRDL), Univ. Bretagne-Sud, EA 4250, LIMATB, F-56100 Lorient, \\ France \\ ${ }^{3}$ Department of Mathematical Physics, Ural Federal University, 51, Prospekt Lenina, Ekaterinburg 620083, \\ Russia \\ $†$ Corresponding author: kuzhir@unice.fr
}

This work is focused on the modelling of the shear and normal stresses in fibre suspensions that are subjected to a simple shear flow in the presence of short-range lubrication forces, van der Waals and electrostatic forces, as well as solid friction forces between fibres. All these forces are weighed by the contact probability. The theory is developed for attractive fibres with van der Waals interaction dominating over electrostatic repulsion. The model predicts a simple Bingham law for both the shear stress and the first normal stress difference with the apparent shear and normal yield stresses proportional, respectively, to the second and the third power of particle volume fraction. The model is applied to the experimental data of Rakatekar et al. Adv. Mater 21, 874-878 (2009) and Natale et al. AIChE J. 60, 1476-1487 (2014) on the suspensions of carbon nanotubes dispersed in a Newtonian epoxy resin. It reproduces well the quadratic dependency of the apparent yield stress on particle volume fraction $\left(\sigma_{Y} \propto \phi^{2}\right)$ for average particle aspect ratios $r=160$ and 1200, while it under-predicts the power-law exponent for $r=80$ (always predicting $\phi^{2}$ - behaviour instead of $\phi^{3.2}$ ).

\section{Introduction}

Rigid rod-like particles are known to strengthen significantly the visco-elastic properties of the composite materials (Christensen (1991)). Fibre-reinforced composites have numerous industrial applications ranging from concretes to reinforced plastics broadly used in automobile and aerospace industries. Among different types of rod-like particles, carbon nanotubes (CNT) have various advantages related to their relatively small size, high rigidity and extremely high aspect ratio allowing a strong enhancement of the elastic moduli of the composites (Allaoui et al. (2002)). However, particle aggregation under colloidal interactions lowers their effective aspect ratio and can induce thixotropic effects (Rahatekar et al. (2006), Khalkhal et al. (2011)). The chemical treatment improves the CNT dispersion state, however, it does not completely prevent the adhesive contacts between nanotubes because, being dispersed in a Newtonian solvent, the CNT suspensions still show a shear thinning behaviour (Song \& Youn (2005), Fan \& Advani (2007)). Therefore, a clearer understanding of the role of colloidal interactions between nanotubes is extremely important for the proper design of the CNT composites. 
If the hydrodynamic interactions in fibre suspensions have received much attention during the last decades (see reviews by Larson (1999), Petrie (1999), Férec \& Ausias (2015)), the colloidal and contact interactions have received less attention. Shear thinning behaviour related to non hydrodynamic interactions have been observed experimentally for suspensions composed of different types of fibres, like ceramic (Bergström (1998)), polyamide (Mongruel \& Cloitre (1999), Chaouche \& Koch (2001)), wollastonite (Mueller et al. (2010)), nontronite clay (Michot et al. (2009)), colloidal hematite (Solomon \& Boger (1998)), multiwall CNT (Khalkhal et al. (2011)). Some colloidal suspensions experienced strong flocculation (Wierenga et al. (1998), Wolf et al. (2007)) resulting in a yield stress. In particular, Saarinen et. al. (2014) has carefully studied the flows and the microstructure of the micro-fibrillated cellulose suspensions near the yield point using the optical coherence tomography coupled with the cylindrical Couette rheometry. The authors have revealed a strongly flocculated state of the suspension depending on the ionic strength and on the shear rate and leading to heterogeneous flows with pronounced wall slip. Yield behaviour has also been observed for the non-Brownian pulp suspensions (Bennington et al. (1990), Cui \& Grace (2007)) and is conventionally explained in terms of the combination between fibre flexibility and inter-fibre friction. Chaouche \& Koch (2001) have measured the adhesive force acting between two nylon fibres of a diameter ranging between 14 and $28 \mu \mathrm{m}$ and have found the values ranging between $10^{-8}$ and $10^{-6} \mathrm{~N}$. To the best of our knowledge, this is the single work quantifying the colloidal forces in non Brownian fibre suspensions and underlying importance of these forces on the shear thinning behaviour.

Theoretical modelling of non-Newtonian effects in fibre suspensions concerns mostly flexible fibres, whose shear thinning behaviour is governed by the ratio of the fibre elastic modulus to the applied shear stress and the yield stress appears as a result of the solid friction between the packed fibres (Bennington et al. (1990), Toll \& Månson (1994), Servais et al. (1999)). In a series of papers, Klingenberg and co-workers have reproduced the rheology of flexible fibre suspensions by particle-level numerical simulations (see for instance Schmid \& Klingenberg (2000); Switzer \& Klingenberg (2004)). In addition to the solid friction, they have included an attractive force between fibres. A phenomenological expression have been proposed for this force: it was supposed to be exponentially decaying with the separation between fibres, linear in shear rate and independent of the mutual orientation of fibres. The authors concluded on a weak effect of this force on the suspension rheology and maintained the interplay between the solid friction and the fibre entanglement as the main mechanism governing the non-Newtonian behaviour.

However, the above-mentioned scenario cannot explain shear thinning effects in suspensions of rigid fibres dispersed in a Newtonian solvent (Bergström (1998), Mongruel \& Cloitre (1999), Chaouche \& Koch (2001)). The theoretical modelling of these effects has started quite recently. Natale et al. (2014) have extended the models of Férec et al. (2009) and Djalili-Moghaddam \& Toll (2005) to non-Newtonian lubrication contacts between fibres. They mimicked the resultant hydrodynamic and non-hydrodynamic interactions between fibres by a power-law lubrication law with shear-thinning index and consistency taken as adjustable parameters. The interaction force has been weighed by the contact probability and 
was taken into account in the stress tensor of the suspension. When the shear thinning index is set to 0 , the interaction force between fibres becomes independent of shear rate and leads to the yield stress in the fibre suspension (Férec et al. (2015)). Another approach, elaborated by Ma et al. (2008), consists of considering a series of fibre populations having different degrees of aggregation characterized by phenomenological aggregation and destruction times. The probability density of finding a fibre in a given population with a prescribed orientation was found by a solution of the properly formulated Fokker-Planck equation, while the stress tensor was calculated by averaging over all populations and orientations. Both models gave a satisfactory correspondence with experiments reproducing a strong shear thinning behaviour of CNT suspensions. However, the models remain mainly phenomenological without considering adhesive interaction potential. The first model captures the shear thinning by a manually introduced power-law exponent of the non-linear lubrication law. The second model makes use of a phenomenological rotary diffusivity of particles belonging to each population. As a consequence, the model predicts progressive particle alignment and a viscosity decrease with growing Péclet number. A more detailed study of the dynamics of aggregates composed of fibres has been recently carried out by Abisset-Chavanne et al. (2013) and AbissetChavanne et al. (2015). However, the colloidal forces have not still been taken into consideration and the effect of the aggregates on the suspension rheology is not reported.

Since the role of the adhesive contacts on the rheology of rigid fibre suspensions remains poorly understood, we decided to construct a theoretical model considering micromechanics of the fibre-fibre contacts with colloidal van der Waals and electrostatic interactions. The basic idea consists in calculation of the resulting colloidal force between two contacting fibres, weighing this force by the probability of inter-particle contact and introducing the appropriate terms into the equation of motion and into the stress tensor. In theory, the shear thinning behaviour is not necessarily connected to particle aggregation. Nonhydrodynamic inter-particle forces (scaling non-linearly with shear rate) will inevitably lead to non-linear shear stress versus shear rate dependency even if the particles still remain perfectly dispersed. Clearly, the van der Waals interactions promote the aggregation, which is expected to strongly affect the stress level and the orientation state of the fibres. However, it is important to inspect these both effects separately in order to gain a clearer insight into the physics behind them. Therefore, in this work we focus only on effect of the colloidal interactions on the fibre orientation and on the suspension stress without considering aggregation. We consider only the case of overall attractive interaction, i.e. van der Waals attraction is supposed to dominate over electrostatic repulsion.

In the next $\S 2$, we present our theoretical model. In $\S 3$, we compare our model with the experimental results of Natale et al. (2014) on the shear viscosity and the first normal stress difference of CNT suspensions. Finally, conclusions and perspectives are outlined in $\S 4$.

\section{Theory}

Under shear flow, the fibres enter into a close contact with their neighbours, and strong short-range forces may appear. At the beginning, in $\$ 2.1$, we will present the main 
assumptions of the model, then, in $\S 2.2$, we will find the expressions for the interaction forces between fibres; we will also integrate these forces over the contact probability and obtain the mean interaction force. In $\S 2.3$, we will find the fibre orientation distribution, and finally in $\S 2.4$, we will proceed to the calculation of the shear and normal stresses.

\subsection{Main assumptions}

Let us consider a steady state simple shear flow of a suspension of perfectly rigid fibres dispersed in a Newtonian solvent of viscosity $\eta_{0}$. The flow is characterized by a shear rate $\dot{\gamma}$. The Cartesian coordinate frame is chosen in such a way that the axes 1,2 and 3 correspond to the flow, velocity gradient and vorticity directions, respectively, as depicted in figure 1 .

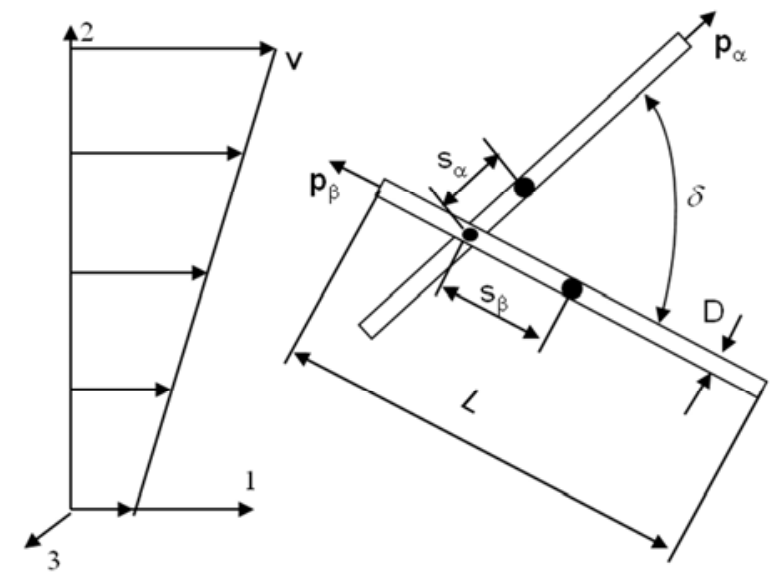

Figure 1. Sketch of the contact between two fibres

To get a qualitative picture of the effect of colloidal forces on the rheology of fibre suspensions, we will discard some secondary details of the experimental system and introduce the following assumptions:

1. According to characterizations performed by Khalkhal et al. (2011), the CNT particles showed some distribution of their length and diameter. However, for the sake of simplicity, we shall consider the fibres to be monodisperse, thus all having the same length $L$, the same diameter $D$ and the same aspect ratio $r=L / D$. For definiteness, the average values $D \approx 16 \mathrm{~nm}$ of the fibre diameter and $r \approx 160$ of the aspect ratio will be used.

2. The relative importance of the Brownian motion with respect to hydrodynamic interactions is described by the Péclet number [Larson (1999)]: $P e \approx \pi \eta_{0} L^{3} \dot{\gamma} /\left[3 k_{B} T(\ln r-0.8)\right]$, where $\eta_{0}=12.3 \mathrm{~Pa} \cdot \mathrm{s}$ is the suspending fluid viscosity, $k_{B} \approx 1.38 \cdot 10^{-23} \mathrm{~J} / \mathrm{K}$ is the Boltzmann constant, $T \approx 300 \mathrm{~K}$ is the absolute temperature. In the range of the shear rates, $10^{-2} \leq \dot{\gamma} \leq 10^{2} \mathrm{~s}^{-1}$, used in experiments of Natale et al. (2014), the Péclet number varies in the range $13 \leq P e \leq 1.3 \cdot 10^{5}$. Such high values of the Péclet number allow us to consider CNT as non-Brownian fibers. 
3. According to estimations of Khalkhal et al. (2011) (who characterized the CNTs used in experiments of Natale et al. (2014)), the CNTs are expected to be very rigid to the bending within the range of the applied shear stresses. However, the electron microscopy reveals some bending of fibres likely because of some local defects. The effect of such defects on CNT orientation state under flow has been recently studied by Natale et al. (2015). Keeping in mind the possible importance of the CNT structural defects on the suspension rheology, we prefer to focus on the effect of colloidal interactions. Thus, at the first approximation, we consider the fibres to be perfectly rigid and straight.

4. We consider both van der Waals attraction and electrostatic repulsion between fibres through Derjaguin-Landau-Verwey-Overbeek (DLVO) theory adapted to rod-like particles. The electrostatic repulsion is considered at either the limit of a weak electrostatic potential $\Psi$ on the fibre surface ( $e \Psi \ll k_{B} T$, with $e$ - elementary charge) or the thin electric double layer limit ( $\kappa^{-1} \ll D$, with $\kappa^{-1}$ - Debye length). At these conditions, verified for non-stabilized $\mathrm{CNT} /$ epoxy composites, the electric double layer does not significantly increase the effective hydrodynamic diameter of fibres and, consequently does not lead to strong excluded volume effects occurring in strongly repulsive colloids. The resulting colloidal interaction is attractive and is expected to lead to small separations, $h \ll D$, between fibres in contact points, as will be proved in $\S 3$.

5. According to the work of Petrich \& Koch (1998), at small separations $h$, the energy dissipation within the inter-fibre gaps is governed by both a hydrodynamic lubrication and a tangential solid-like friction. The latter is described by the classical Amontons-Coulomb friction law:

$$
F_{f r}=\mu F_{\perp}
$$

where $F_{\perp}$ is the normal component of the resulting attractive force between particles and $\mu$ is the friction coefficient.

6. We suppose only sliding contacts between particles, i.e. the fibres do not stick to each other but always slide over each other in all contact points. The sliding contacts are expected to prevent the particles from aggregation, and we neglect any possible aggregation. This assumption implies an affine translational motion of fibres, as we shall prove in $\S 2.2$. The present hypothesis will be further discussed in $\S 2.2$ and $\S 4$.

7. The interactions between fibres and walls are neglected. This assumption should hold relatively well for the high ratio of the rheometer gap to the CNT length. To perform their experiments, Natale et al. (2014) used a cone-and-plate flow geometry, for which the gap at the centre of the cone is found to be 25 times larger than the longest CNT.

\subsection{Inter-particle forces}

A sketch of a mutual position of two contacting fibres (marked as $\alpha$ and $\beta$ ) is shown in

figure 1 . The orientation of both fibres is described by unit vectors $\mathbf{p}^{\alpha}$ and $\mathbf{p}^{\beta}$, while the 
distance between the contact point and the centre of mass of each fibre is denoted by $s_{\alpha}$ and $s_{\beta}$. According to the assumptions (4) and (5), the following short range forces act between the contacting fibres: a lubrication force $\mathbf{F}_{l u b}$, an attractive van der Waals force, $\mathbf{F}_{v d W}$, a repulsive electrostatic force $\mathbf{F}_{e l}$ and a tangential friction force $\mathbf{F}_{f r}$. It can be shown that the normal component of the van der Waals and electrostatic forces generates only the odd moments of the orientation distribution function $(\langle\mathbf{p}\rangle,\langle\mathbf{p p p}\rangle, \ldots)$ in the stress tensor and in the equation of fibre rotation. Since these moments are zero because of symmetry reasons, the normal component of $\mathbf{F}_{v d W}$ and $\mathbf{F}_{e l}$ does not give any contribution to the stress and the orientation state, of course, only under assumption (6) of perfect sliding without aggregation. Analysis shows that the tangential component of $\mathbf{F}_{v d W}$ and $\mathbf{F}_{e l}$ appears to be negligible relatively to the friction force $\mathbf{F}_{f r}$ even at low friction coefficients. Therefore, only the lubrication and the solid friction force will give leading order contributions to the suspension stress.

The lubrication force may depend not only on the separation between fibre surfaces but also on microscopic details of the lubricated contacts, such as surface roughness and possible deformation of the contact area. Férec et al. (2009) have proposed a simple scaling $\mathbf{F}_{\text {lub }} \propto \eta_{0} D \mathbf{u}$ discarding these details, with $\mathbf{u}$ being the relative velocity of the approach of two fibres. This scaling neglects the effect of the mutual orientation of fibres. Intuitively, when the fibres get more aligned with respect to each other, the contact area increases inversely proportionally to the sine of the angle $\delta$ between fibres (figure 1). The lubrication force is roughly proportional to the contact area and is expected to be inversely proportional to $\sin \delta=\left|\mathbf{p}^{\alpha} \times \mathbf{p}^{\beta}\right|$, similarly to the case of the classical lubrication between two perfectly smooth cylinders without elastic deformations [Yamane et al. (1994)]. Thus, the expression for the lubrication force reads:

$$
\mathbf{F}_{\text {lub }}=\frac{k \eta_{0} D \mathbf{u}}{\left|\mathbf{p}^{\alpha} \times \mathbf{p}^{\beta}\right|}
$$

where $k$ is a dimensionless constant depending on the microscopic details of the lubricated contact. This constant is taken as a free parameter of the model. According to the assumption (5), the solid friction force [Eq. (1)] can be written in the following vector form:

$$
\mathbf{F}_{f r}=\mu F_{\perp} \frac{\mathbf{u}_{\alpha \beta}}{\left|\mathbf{u}_{\alpha \beta}\right|}
$$

where $\mathbf{u}_{\alpha \beta}$ is the projection of the velocity of the relative motion of the contact points of the neighbouring fibres " $\alpha$ " and " $\beta$ " onto the $\alpha \beta$-plane formed by both fibres.

According to the DLVO theory, the resulting energy $U$ of colloidal interactions between particles is the sum of the energies $U_{v d W}$ and $U_{e l}$ of van der Waals and electrostatic interactions, respectively. Grossberg \& Khohlov (1981), van der Schoot \& Odjik (1992) have proposed a theoretical expression for $U_{v d W}$ for rod-like particles with $r \gg 1$ and at small separations $h \ll D$. On the other hand, Brenner \& Parsegian (1974) have derived the 
expression for $U_{e l}$ for a weak electrostatic repulsion $\left[e \Psi \ll k_{B} T\right.$ or $\kappa^{-1} \ll D$, cf. assumption (4)] at $r \gg 1$ and any $h>0$. The resulting energy and the normal component of the colloidal forces are given by the following expressions:

$$
\begin{gathered}
U=U_{v d W}+U_{e l}=-\frac{A}{12} \frac{D}{h\left|\mathbf{p}^{\alpha} \times \mathbf{p}^{\beta}\right|}+\frac{v^{2}}{4 \varepsilon_{0} \varepsilon \kappa} \frac{\exp [-\kappa(D+h)]}{\left|\mathbf{p}^{\alpha} \times \mathbf{p}^{\beta}\right|} \\
F_{\perp}=-\left(\frac{\partial U}{\partial h}\right)_{\delta}=\frac{F}{\left|\mathbf{p}^{\alpha} \times \mathbf{p}^{\beta}\right|} \\
F=F_{v d w}+F_{e l}=\frac{A}{12} \frac{D}{h^{2}}-\frac{v^{2}}{4 \varepsilon_{0} \varepsilon} \exp [-\kappa(D+h)]>0
\end{gathered}
$$

where $A$ is the Hamaker constant; $\varepsilon_{0}=8.85 \cdot 10^{-12} \mathrm{~F} / \mathrm{m}$ - the dielectric permittivity of vacuum, $\varepsilon$ - the relative dielectric permittivity of the suspending fluid, $\kappa$ - the inverse Debye length, $v$ linear electric charge density on the fibre surface, $F$ is a typical value of the resulting colloidal force when both fibres are orthogonal to each other. Only positive values of $F$ results in nonzero solid friction force between fibres. The condition $F>0$ is automatically verified under the used assumption on weakness of electrostatic interactions. Notice that equations (2) and (4) are only valid if the mutual orientation of both fibres is such that $\sin \delta>1 / r$ (Grossberg \& Khohlov (1981), van der Schoot \& Odjik (1992), Brenner \& Parsegian (1974)). This condition will be checked in $\S 2.5$.

\subsection{Averaging of the interaction forces over the contact probability}

In order to get the total force acting on a given fibre by neighbouring fibres, first, we need to express the velocities $\mathbf{u}$ and $\mathbf{u}_{\alpha \beta}$ through the orientation vectors of the fibres. The position of the centre of mass of both fibres is described by the radius vectors $\mathbf{r}_{\alpha}$ and $\mathbf{r}_{\beta}$. The expressions for the vector $\Delta \mathbf{r}$ connecting the nearest points of the axes of both fibres and for the relative velocity $\mathbf{u}$ of the fibres' approach (or rather relative velocity of these two points) are obtained from geometrical and kinematic considerations, respectively:

$$
\begin{aligned}
& \Delta \mathbf{r}=\left(\mathbf{r}_{\beta}+s_{\beta} \mathbf{p}^{\beta}\right)-\left(\mathbf{r}_{\alpha}+s_{\alpha} \mathbf{p}^{\alpha}\right), \\
& \mathbf{u}=\Delta \dot{\mathbf{r}}=\left(\dot{\mathbf{r}}_{\beta}+s_{\beta} \dot{\mathbf{p}}^{\beta}\right)-\left(\dot{\mathbf{r}}_{\alpha}+s_{\alpha} \dot{\mathbf{p}}^{\alpha}\right),
\end{aligned}
$$

where $\dot{\mathbf{r}}_{\alpha}=\boldsymbol{\xi} \cdot \mathbf{r}_{\alpha}$ and $\dot{\mathbf{r}}_{\beta}=\boldsymbol{\xi} \cdot \mathbf{r}_{\beta}$ are the velocities of the centre of mass of both fibres, $\boldsymbol{\xi}=\boldsymbol{\omega}+\boldsymbol{\gamma}=\nabla \mathbf{v}, \quad \boldsymbol{\omega}=(1 / 2)\left(\nabla \mathbf{v}-\nabla \mathbf{v}^{T}\right)$ and $\boldsymbol{\gamma}=(1 / 2)\left(\nabla \mathbf{v}+\nabla \mathbf{v}^{T}\right)$ are the velocity gradient, vorticity and rate-of-strain tensors, and the vector $\mathbf{v}$ describes the linear velocity field in the fibre suspension. From now, we shall use the component notation for the vectors and tensors and adopt the convention of the summation over the repeated indices. The rotation speed of the fibres can be estimated in the dilute-limit approach using the Jeffery formula (Jeffery (1922), Larson (1999)): 


$$
\dot{p}_{i}=\omega_{i k} p_{k}+\lambda\left(\gamma_{i k} p_{k}-p_{i} p_{k} p_{l} \gamma_{k l}\right)
$$

where the superscripts " $\alpha$ " and " $\beta$ " over $\mathbf{p}$ are omitted for brevity; $\lambda=\left(r_{e}^{2}-1\right) /\left(r_{e}^{2}+1\right)$ is the form-factor of the fibres and $r_{e}=1.24 r / \mathrm{ln}^{1 / 2} r$ is the equivalent aspect ratio of the cylindrical particles (Brenner (1974)). Combination of equations (5) and (6) gives us the following expression for the relative velocity of the approach of two fibres:

$u_{l}=\xi_{l m} \Delta r_{m}-(1-\lambda)\left(s_{\beta} \gamma_{l m} p_{m}{ }^{\beta}-s_{\alpha} \gamma_{l m} p_{m}{ }^{\alpha}\right)-\lambda\left(s_{\beta} p_{l}^{\beta} p_{m}{ }^{\beta} p_{s}{ }^{\beta} \gamma_{m s}-s_{\alpha} p_{l}^{\alpha} p_{m}{ }^{\alpha} p_{s}^{\alpha} \gamma_{m s}\right)$

Here $\Delta \mathbf{r}$ can be expressed through the distance between the nearest points of the fibre axes $\Delta r$ as $\Delta \mathbf{r}= \pm \Delta r\left(\mathbf{p}^{\alpha} \times \mathbf{p}^{\beta}\right) /\left|\mathbf{p}^{\alpha} \times \mathbf{p}^{\beta}\right|$. As a consequence, the first term of (7) will always result in odd moments of the orientation distribution function $(\langle\mathbf{p}\rangle,\langle\mathbf{p p p}\rangle, \ldots)$, which are all zero. For this reason, this term will be hereinafter omitted. In the limit of high aspect ratio fibres, $r \gg 1$ and $\lambda \approx 1-2 / r_{e}^{2}$, the second and the third terms of (7) are of the order of magnitude $\dot{\gamma} L / r^{2}$ and $\dot{\gamma} L$ respectively. The second term can be therefore neglected and equation (7) will take the following form:

$$
u_{l} \approx s_{\alpha} p_{l}^{\alpha} p_{m}^{\alpha} p_{s}^{\alpha} \gamma_{m s}-s_{\beta} p_{l}^{\beta} p_{m}^{\beta} p_{s}^{\beta} \gamma_{m s}
$$

Note that, within the assumption $r \gg 1(\lambda \approx 1)$, the first and the second terms of ( 8 ) represent the components of the relative velocity along the fibres " $\alpha$ " and " $\beta$ ", respectively. Therefore, the vector $\mathbf{u}$ lies in the plane formed by both fibres and the component $\mathbf{u}_{\alpha \beta} \approx \mathbf{u}$. Thus, we will not make any distinction between the magnitudes $\mathbf{u}$ and $\mathbf{u}_{\alpha \beta}$ appearing in (2) and (3).

The relationship (8) is similar to the one derived by Djalili-Moghaddam \& Toll (1995) but differs from that derived by Férec et al. (2009) who have used the co-rotational frame. We argue that material objectivity should be naturally satisfied in the classical Cartesian coordinate frame because we derive the orientation and stress tensors from the microscopic approach (by ensemble average of the local orientation and stress fields) without using any phenomenological constitutive relationships. Thus, it is convenient to use the classical Cartesian coordinate frame.

Let us now define the probability $\mathrm{d} P$ of fibre contact, as the number of fibres $\beta$ intersecting the segment of the test fibre $\alpha$ at the angle corresponding to the orientation vector $\mathbf{p}^{\beta}$, with the contact happening within the segment length $d s_{\alpha}$ and $d s_{\beta}$ (Doi \& Edwards (1986), Férec et al. (2009)):

$$
d P=2 n D\left|\mathbf{p}^{\alpha} \times \mathbf{p}^{\beta}\right| d s_{\alpha} d s_{\beta} \psi_{\beta} d \mathbf{p}^{\beta}
$$

where $n$ is the number fraction of the fibres and $\psi_{\beta}$ is the angular distribution function of the neighbouring fibres $\beta$. 
Thus, all the quantities related to the fibre contacts will be averaged first over the contact probability (this averaging will be denoted by $\langle\ldots\rangle_{P}$ ), and then over the orientation of the test fibre. For example, the average lubrication and solid friction forces acting on the test fibre $\alpha$ are defined as: $\left\langle\mathbf{F}_{\text {lub }}\right\rangle_{P}=\int \mathbf{F}_{\text {lub }} d P$ and $\left\langle\mathbf{F}_{f r}\right\rangle_{P}=\int \mathbf{F}_{f r} d P$. Since $\mathbf{F}_{\text {lub }} \propto \mathbf{u}$ and $\mathbf{F}_{f r} \propto \mathbf{u} /|\mathbf{u}|$ are odd functions of $s_{\alpha}$ and $s_{\beta}$ (cf. equation (8) for $\mathbf{u}$ ), their integration over the lengths of both fibres gives zero. We prove therefore that the average force exerted by the neighbouring fibres to the test fibre is zero. In this case, the fibres displace affinely with the flow, i.e. their centre of mass displaces with the velocity of the suspending liquid, as already stated in assumption (4) of $\$ 2.1$. We should notice however that this assumption holds only in the case of perfect sliding contacts when all the fibres slide along each other with a relative velocity given by (8). In reality, for some contact points, the tangential friction force may appear to be less than the product $\mu F_{\perp}$. The fibres will stick to each other in these points and their translational motion will not more be affine. At strong enough attractive colloidal force, $F_{\perp}$, each fibre can have several sticking contacts promoting appearance of a fibre network. As already mentioned, we ignore this scenario in the present work.

\subsection{Equation of angular motion of the test fibre}

This equation describes the temporal evolution of the unit vector $\mathbf{p}^{\alpha}$ and takes the following form in the presence of non-hydrodynamic torques (Pokrovskiy (1978)):

$$
\dot{p}_{i}^{\alpha}=\omega_{i k} p_{k}^{\alpha}+\lambda\left(\gamma_{i k} p_{k}^{\alpha}-p_{i}^{\alpha} p_{k}^{\alpha} p_{l}^{\alpha} \gamma_{k l}\right)+\frac{1}{\eta_{0} \beta f^{\perp} V_{f}}\left\langle T_{i k} p_{k}^{\alpha}\right\rangle_{P}
$$

where $V_{f}$ is the fibre volume, the form factors $\beta$ and $f^{\perp}$ are defined below equation (15); $T_{i k}$ is the interaction torque tensor related to the total force $\mathbf{F}=\mathbf{F}_{\mathrm{lub}}+\mathbf{F}_{f r}$ acting on a given contact point of the test fibre $\alpha$; this tensor is given by the following expression:

$$
T_{i k}=s_{\alpha}\left(p_{k}^{\alpha} F_{i}-p_{i}^{\alpha} F_{k}\right)
$$

Combining (2), (3), (4.2), (8) and (11) together, we obtain the following expression for the magnitude $T_{i k} p_{k}{ }^{\alpha}$ appearing in the last term of (10):

$T_{i k} p_{k}^{\alpha}=s_{\alpha}\left(F_{i}-p_{i}^{\alpha} p_{k}^{\alpha} F_{k}\right)=-\left(\frac{k \eta_{0} D s_{\alpha} s_{\beta}}{\left|\mathbf{p}^{\alpha} \times \mathbf{p}^{\beta}\right|}+\frac{\mu F s_{\alpha} s_{\beta}}{|\mathbf{u}|\left|\mathbf{p}^{\alpha} \times \mathbf{p}^{\beta}\right|}\right)\left(p_{i}^{\beta} p_{k}{ }^{\beta} p_{l}^{\beta} \gamma_{k l}-p_{i}^{\alpha} p_{k}{ }^{\alpha} p_{k}{ }^{\beta} p_{l}^{\beta} p_{m}{ }^{\beta} \gamma_{l m}\right)$

where $F$ is the colloidal force given in (4.3).

Since the magnitude $T_{i k} p_{k}{ }^{\alpha}$ is an odd function of $s_{\alpha}$ and $s_{\beta}$, its integration over the length of both fibres will give zero. Besides this, the averaging over the orientations of the neighbouring fibres $\beta$ will results in the odd moment $\langle\mathbf{p p p}\rangle$ of the orientation distribution function, which is zero because of symmetry reasons. The equation of the angular motion of the test fibre reduces therefore to the classical Jeffery equation (6). Note that if, instead of (6) 
we use equation (10) in (5.2) while determining the relative velocity $\mathbf{u}$, we will still arrive at $\left\langle T_{i k} p_{k}{ }^{\alpha}\right\rangle_{P}=0$ and the fibre angular motion will remain unaltered by the contact forces. At the first glance, this result seems to contradict to the statement that fibre orientation should be strongly affected by particle-particle interactions. However, equation (6) describes only the deterministic motion of fibres, apparently not influenced by short-range interactions. The stochastic motion is expected to be affected by these interactions because the rotary diffusivity should be an increasing function of the short-range forces resulting in a more random orientation distribution with increasing interactions.

\subsection{Orientation distribution}

The fibre orientation distribution function is given by the Fokker-Planck equation which takes the similar form as for the fibre suspension without short-range interactions (Larson (1999)). We assume that the rotary diffusion of fibres is mostly governed by hydrodynamic and short-range contact interactions and suppose the diffusivity to be proportional to the fibre volume fraction $\phi$ and the shear rate $\dot{\gamma}$ :

$$
D_{r}=C \phi \dot{\gamma}
$$

where $C$ is a dimensionless constant which can depend on the fibre aspect ratio and is taken as an adjustable parameter of the model. The rotary diffusivity given by (13) is similar to the one proposed by Folgar \& Tucker (1984), $D_{r}=C_{I} \dot{\gamma}$. In our model the Folgar \& Tucker's interaction constant is chosen to be proportional to particle concentration, $C_{I}=C \phi$, and therefore accounts for pair interactions.

We assume a weak diffusion limit, $D_{r} r_{e}^{3} / \dot{\gamma}=C \phi r_{e}^{3} \ll 1$ at $r \gg 1$, described in details by Leal \& Hinch (1971) and Hinch \& Leal (1972). These authors have solved the FokkerPlanck equation for the simple shear flow and derived analytical expressions for the even moments $\langle\mathbf{p p}\rangle$ and $\langle\mathbf{p p p p}\rangle_{\text {of }}$ the orientation distribution function. We write down the formulas (including only the leading terms) for the three goniometric factors that will be used in $\S 2.4$ for the stress calculation:

$$
\begin{aligned}
& \left\langle p_{1}^{2} p_{2}^{2}\right\rangle \approx \frac{a}{r_{e}}=\frac{c \ln ^{1 / 2} r}{r} \\
& \left\langle p_{1}^{3} p_{2}\right\rangle-\left\langle p_{1} p_{2}^{3}\right\rangle \approx \frac{1}{4} \frac{D_{r} r_{e}^{2}}{\dot{\gamma}}=\frac{1}{4} C b \phi \frac{r^{2}}{\ln r} \\
& \left\langle p_{1} p_{2}{ }^{3}\right\rangle-\left\langle p_{1} p_{2} p_{3}{ }^{2}\right\rangle \approx-\frac{5}{2} \frac{D_{r}}{\dot{\gamma}}=-\frac{5}{2} C \phi
\end{aligned}
$$

where we have used the relationship $r_{e}=1.24 r / \ln ^{1 / 2} r$ for the equivalent aspect ratio; the numerical constants $a, b$ and $c$ come from the integration of the orientation distribution function of Leal \& Hinch (1971) and take the following values: $a=0.315, b=1.54$ and 
$c=a / b^{1 / 2}=0.254$. Notice that the factor $C \phi$ describes eventual effects of the short-ranged hydrodynamic and contact interactions between fibres on the rotary diffusivity and therefore on the fibre orientation state. As is seen from (14), only the two last goniometric factors (14.2) and (14.3) are affected by these interactions in the low diffusion limit $C \phi r_{e}^{3} \ll 1$.

Having defined the orientation distribution, we can now check the condition $\sin \delta>1 / r$ of validity of equations (2) and (4). According to Férec et al. (2009), the mean value of $\sin \delta$ can be estimated as $\langle\sin \delta\rangle \approx(3 \pi / 8)(1-\langle\mathbf{p p}\rangle\langle\mathbf{p p}\rangle)$. Following the work of Leal \& Hinch (1971), we estimate firstly the product $\langle\mathbf{p p}\rangle\langle\mathbf{p p}\rangle=4.844 / r+O\left(1 / r^{2}\right)$ and secondly the sine of the angle $\delta:\langle\sin \delta\rangle \approx 5.71 / r$. As we see, the condition $\sin \delta>1 / r$ is verified, at least for the average orientation state.

\subsection{Shear and normal stresses}

It can be easily shown that in the absence of external torques acting on fibres, the particle stress is entirely defined by the longitudinal stress generated on fibres. This proof is briefly presented in Appendix A. The general expression for the stress tensor reads (Brenner (1974), Pokrovskiy (1978)):

$$
\begin{aligned}
& \sigma_{i k}=-p \delta_{i k}+\eta_{0} \gamma_{i k}+n V_{f} \eta_{0}\left\{4 \gamma_{i k}+\frac{\beta f^{\|}}{2}\left[\left\langle p_{i} p_{k} p_{l} p_{m}\right\rangle-\frac{1}{3} \delta_{i k}\left\langle p_{l} p_{m}\right\rangle\right] \gamma_{l m}\right\}+ \\
& +n V_{f} \beta f^{\perp} \eta_{0} D_{r}\left[3\left\langle p_{i} p_{k}\right\rangle-\delta_{i k}\right]+n\left\langle p_{i} p_{k} p_{l} \varphi_{l}\right\rangle \\
& n\left\langle p_{i} p_{k} p_{l} \varphi_{l}\right\rangle=n\left\langle p_{i} p_{k} p_{l} \int s_{\alpha} F_{l} d P\right\rangle=2 n^{2} D \int_{\mathbf{p}^{\alpha}} \psi_{\alpha} d \mathbf{p}^{\alpha} \int_{\mathbf{p}^{\beta}} \psi_{\beta} d \mathbf{p}^{\beta} \int_{-L / 2}^{L / 2} s_{\alpha} d s_{\alpha} \int_{-L / 2}^{L / 2}\left|\mathbf{p}^{\alpha} \times \mathbf{p}^{\beta}\right| p_{i}^{\alpha} p_{k}^{\alpha} p_{l}^{\alpha} F_{l} d s_{\beta}
\end{aligned}
$$

where $p$ is the hydrostatic pressure, $n$ is the number fraction of fibres, $F_{l}$ is the " $l$ "-component of the total short-range force $\mathbf{F}=\mathbf{F}_{\text {lub }}+\mathbf{F}_{f r}$ acting between fibres; $\psi_{\alpha}$ is the orientation distribution function for the test fibre $\alpha ; \delta_{i k}$ is the delta Kronecker; $\beta=4 r^{2} \varepsilon / 3$, $f^{\perp}=(1+0.64 \varepsilon) /(1-0.5 \varepsilon), \quad f^{\|}=(1+0.64 \varepsilon) /(1-1.5 \varepsilon)$ and $\varepsilon=1 / \ln (2 r)$ are the form factors estimated with the help of the slender body theory of Batchelor (1970) in the dilute limit. The first two terms of (15.1) represent the stress contribution from the suspending liquid, the third term is the hydrodynamic stress generated by the fibres, the fourth term comes from stochastic fluctuations of the fibre orientation (so-called diffusion stress) and the last term comes from short-ranged forces acting between fibres. Equation (15.1) is the same as proposed by Brenner (1974) and Pokrovskiy (1978) except for the supplementary term $n\left\langle p_{i} p_{k} p_{l} \varphi_{l}\right\rangle$, in which we introduced the short-range force $\mathbf{F}=\mathbf{F}_{\text {lub }}+\mathbf{F}_{f r}$ between fibres. Note that, in its general form, this stress term differs from the term $n\left\langle p_{i} \varphi_{k}\right\rangle \propto n L\left\langle p_{i} F_{k}\right\rangle$ used by Toll \& Månson (1994), Djalili-Moghaddam \& Toll (2005) and Férec et al. (2009). These authors considered the interparticle stress to be proportional to the dyadic product of the position vector $s_{\alpha} \mathbf{p}^{\alpha}$ and the 
inter-particle force $\mathbf{F}$, while we take only the longitudinal component of the inter-particle stress $s_{\alpha} \mathbf{p}^{\alpha} \mathbf{F}$ because the transverse components are compensated by the hydrodynamic moment acting on fibres (cf. equations (A3)-(A4) in Appendix A).

Strictly speaking, the integration in (15.2) cannot be performed analytically for the term including the friction force. This is because the absolute value $|\mathbf{u}|$ of the relative velocity depends on $\mathbf{p}^{\alpha}, \mathbf{p}^{\beta}, s_{\alpha}$ and $s_{\beta}$ and intervenes into the denominator of the expression (3) for the friction force. However this difficulty can be overcome if we perform the pre-averaging of the magnitude $|\mathbf{u}|$ both over the contact probability and over the orientation of the test fibre: $|\mathbf{u}| \rightarrow\langle|\mathbf{u}|\rangle$, where the averaging is performed in a similar way as in (15.2). This kind of preaveraging has been used by Doi \& Edwards (1986) for determining of the mean rotary diffusivity of polymer molecules in the frame of the tube model. The magnitude $|\mathbf{u}|$ is obtained as the square root of the dot product $\mathbf{u}^{2}=u_{l} u_{l}$, with $u_{l}$ given by (8):

$$
\begin{aligned}
& |\mathbf{u}|=\sqrt{\mathbf{u}^{2}}=\sqrt{u_{l} u_{l}}= \\
& \left(s_{\alpha}{ }^{2} p_{k}{ }^{\alpha} p_{l}^{\alpha} p_{m}{ }^{\alpha} p_{n}{ }^{\alpha} \gamma_{k l} \gamma_{m n}-2 s_{\alpha} s_{\beta} p_{i}^{\alpha} p_{k}{ }^{\alpha} p_{l}{ }^{\alpha} p_{i}{ }^{\beta} p_{m}{ }^{\beta} p_{n}{ }^{\beta} \gamma_{k l} \gamma_{m n}+s_{\beta}{ }^{2} p_{k}{ }^{\beta} p_{l}{ }^{\beta} p_{m}{ }^{\beta} p_{n}{ }^{\beta} \gamma_{k l} \gamma_{m n}\right)^{1 / 2}
\end{aligned}
$$

Exact integration of (16) over $\mathbf{p}^{\alpha}, \mathbf{p}^{\beta}, s_{\alpha}$ and $s_{\beta}$ does not allow obtaining analytical results for the suspension stress. However, an approximate expression can be obtained under the following consideration. We estimate that the average value of the relative velocity and of the mean square velocity are of the order of $\langle|\mathbf{u}|\rangle=O\left(L \dot{\gamma} r^{-1} \ln r\right)$ and $\left\langle\mathbf{u}^{2}\right\rangle=O\left(L^{2} \dot{\gamma}^{2} r^{-1}\right)$, respectively. Thus, neglecting the logarithmic term, $\langle|\mathbf{u}|\rangle$ can be related to $\left\langle\mathbf{u}^{2}\right\rangle$ through the following expression: $\langle|\mathbf{u}|\rangle \approx\left\langle\mathbf{u}^{2}\right\rangle /(L \dot{\gamma})$. Performing the averaging, we obtain the following simple expression for $\langle|\mathbf{u}|\rangle$ (the subscript " $\alpha$ " over $\mathbf{p}$ is dropped for brevity):

$$
\begin{gathered}
\langle|\mathbf{u}|\rangle \approx \frac{\left\langle\mathbf{u}^{2}\right\rangle}{L \dot{\gamma}}=\frac{1}{L \dot{\gamma}} \frac{1}{L^{2}} \int_{\mathbf{p}^{\alpha}} \psi_{\alpha} d \mathbf{p}^{\alpha} \int_{\mathbf{p}^{\beta}} \psi_{\beta} d \mathbf{p}^{\beta} \int_{-L / 2-L / 2}^{L / 2} \int_{L^{2 / 2}}^{L} \mathbf{u}^{2} d s_{\alpha} d s_{\beta}=L \dot{\gamma}\langle\hat{u}\rangle \\
\langle\hat{u}\rangle=\frac{1}{6}\left\langle p_{k} p_{l} p_{m} p_{n}\right\rangle \hat{\gamma}_{k l} \hat{\gamma}_{m n}
\end{gathered}
$$

where $\hat{\gamma}_{k l}=\gamma_{k l} / \dot{\gamma}$ is the rate-of-strain tensor normalized by the shear rate $\dot{\gamma}$.

Now we are able to perform the averaging of the last term of the stress tensor. Making use of (2), (3), (4.2), (7) and (17.1) in equations (15.1) and (15.2), the final expression for the stress tensor is written in the following form: 


$$
\begin{aligned}
& \sigma_{i k}=-p \delta_{i k}+2 \eta_{0} \gamma_{i k}+n V_{f} \eta_{0}\left\{4 \gamma_{i k}+\frac{\beta f^{\|}}{2}\left[\left\langle p_{i} p_{k} p_{l} p_{m}\right\rangle-\frac{1}{3} \delta_{i k}\left\langle p_{l} p_{m}\right\rangle\right] \gamma_{l m}\right\}+ \\
& +n V_{f} \beta f^{\perp} \eta_{0} D_{r}\left[3\left\langle p_{i} p_{k}\right\rangle-\delta_{i k}\right]+\frac{1}{6} k n^{2} D^{2} L^{4} \eta_{0}\left\langle p_{i} p_{k} p_{l} p_{m}\right\rangle \gamma_{l m}+\frac{n^{2} D L^{3} \mu F}{6\langle\hat{u}\rangle \dot{\gamma}}\left\langle p_{i} p_{k} p_{l} p_{m}\right\rangle \gamma_{l m}
\end{aligned}
$$

where $F$ and $\hat{u}$ are given by (4.3) and (17.2), respectively and the rotary diffusivity $D_{r}$ should not be confounded with the fibre diameter $D$. The last two terms in (18) stand for the contributions of the short-ranged hydrodynamic and friction force, correspondingly. In the considered weak diffusion limit, $D_{r} r_{e}^{3} / \dot{\gamma} \ll 1$, the diffusion stress is of the order of $n V_{f} \eta_{0} \dot{\gamma}\left(D_{r} r^{2} / \dot{\gamma}\right)^{2}$, while the hydrodynamic stress is of the order of $n V_{f} \eta_{0} \dot{\gamma} r$. The ratio of both is of the order of $\left(D_{r} r^{3} / \dot{\gamma}\right)^{2} / r^{3}$ and appears to be extremely weak for high aspect ratio fibres at $r \gg 1$. Thus, the diffusion stress can be neglected in (18). Note that the orientation tensor $\left\langle\left|\mathbf{p}^{\alpha} \times \mathbf{p}^{\beta}\right| \mathbf{p}^{\alpha} \mathbf{p}^{\alpha} \mathbf{p}^{\alpha} \mathbf{p}^{\alpha}\right\rangle$ does not appear in the stress tensor of our model in contrast to the works of Djalili-Moghaddam \& Toll (1995) and Férec et al. (2009). This is simply because in our model both the lubrication, van der Waals force and electrostatic forces were supposed to be inversely proportional to $\sin \delta=\left|\mathbf{p}^{\alpha} \times \mathbf{p}^{\beta}\right|$ such that the magnitude $\left|\mathbf{p}^{\alpha} \times \mathbf{p}^{\beta}\right|$ worked out during integration over the contact probability $d P \propto\left|\mathbf{p}_{\alpha} \times \mathbf{p}_{\beta}\right|$ (cf. equation (9)).

Now, applying equation (18) for the simple shear flow with $\gamma_{12}=\gamma_{21}=(1 / 2) \dot{\gamma}, \gamma_{i k}=0$ for $i k \neq 12,21$, and using equation (14) for the goniometric factors and (17b) for $\langle\hat{u}\rangle$ (giving $\left.\langle\hat{u}\rangle=(1 / 6)\left\langle p_{1}^{2} p_{2}{ }^{2}\right\rangle\right)$, we arrive at the following expressions for the shear stress and the first normal stress difference in the fibre suspension:

$$
\begin{aligned}
& \sigma_{12}=\eta_{0} \dot{\gamma}\left(1+2 n V_{f}\right)+\frac{1}{2} n V_{f} \eta_{0} \dot{\gamma} \beta f^{\|}\left\langle p_{1}^{2} p_{2}^{2}\right\rangle+\frac{1}{6} k n^{2} D^{2} L^{4} \eta_{0} \dot{\gamma}\left\langle p_{1}^{2} p_{2}^{2}\right\rangle+\frac{n^{2} D L^{3} \mu F}{6\langle\hat{u}\rangle}\left\langle p_{1}^{2} p_{2}{ }^{2}\right\rangle= \\
& =\eta_{0} \dot{\gamma}(1+2 \phi)+\frac{2}{3} c \phi \eta_{0} \dot{\gamma} \frac{r \ln ^{1 / 2}(r) f^{\|}}{\ln (2 r)}+\frac{8}{3 \pi^{2}} c k \phi^{2} \eta_{0} \dot{\gamma} r \ln ^{1 / 2}(r)+\frac{16}{\pi^{2}} \phi^{2} \frac{\mu F}{D^{2}} r
\end{aligned}
$$

$$
\begin{aligned}
& N_{1}=\sigma_{11}-\sigma_{22}=\frac{1}{2} n V_{f} \eta_{0} \dot{\gamma} \beta f^{\|}\left(\left\langle p_{1}^{3} p_{2}\right\rangle-\left\langle p_{1} p_{2}^{3}\right\rangle\right)+\frac{1}{6} k n^{2} D^{2} L^{4} \eta_{0} \dot{\gamma}\left(\left\langle p_{1}^{3} p_{2}\right\rangle-\left\langle p_{1} p_{2}^{3}\right\rangle\right)+ \\
& \frac{n^{2} D L^{3} \mu F}{6\langle\hat{u}\rangle}\left(\left\langle p_{1}^{3} p_{2}\right\rangle-\left\langle p_{1} p_{2}^{3}\right\rangle\right)=\frac{1}{6} C b \phi^{2} \eta_{0} \dot{\gamma} \frac{r^{4} f^{\|}}{\ln (2 r) \ln r}+\frac{2}{3 \pi^{2}} C b k \phi^{3} \eta_{0} \dot{\gamma} \frac{r^{4}}{\ln r}+\frac{4}{\pi^{2}} C \frac{b}{c} \phi^{3} \frac{\mu F}{D^{2}} \frac{r^{4}}{\ln ^{3 / 2} r}
\end{aligned}
$$

where $\phi=n V_{f}$ is the particle volume fraction of the suspension, $b=1.54$ and $c=0.254$. As in suspensions where fibres interact only through hydrodynamic forces, and in the limit $r \gg 1$, the second normal stress difference $N_{2}$ appears to be negligible as compared to $N_{1}$. This comes from the fact that the third goniometric factor (14.3) is negligible with respect to the second 
one (14.2) such that the ratio of the second-to-the first normal stress difference is much smaller than unity:

$$
\frac{N_{2}}{N_{1}}=\frac{\left\langle p_{1} p_{2}^{3}\right\rangle-\left\langle p_{1} p_{2} p_{3}^{2}\right\rangle}{\left\langle p_{1}^{3} p_{2}\right\rangle-\left\langle p_{1} p_{2}^{3}\right\rangle} \approx-\frac{10}{r_{e}^{2}},\left|\frac{N_{2}}{N_{1}}\right| \ll 1
$$

Notice that the last term in (19.1) and (19.2) stands for the solid friction force between fibres and appears to be independent of the shear rate, while the remaining terms are linear in shear rate. This means that both the shear stress and the first normal stress difference follow the Bingham rheological law, $\sigma_{12}=\sigma_{Y}+\eta_{s} \dot{\gamma}$ and $N_{1}=N_{Y}+\eta_{n} \dot{\gamma}$, with the shear and normal viscosities $\eta_{s}$ and $\eta_{n}$ being functions of the particle volume fraction and aspect ratio, while the shear and normal yield stresses are defined by the following expressions:

$$
\begin{gathered}
\sigma_{Y}=\frac{n^{2} D L^{3} \mu F}{6\langle\hat{u}\rangle}\left\langle p_{1}^{2} p_{2}{ }^{2}\right\rangle=\frac{16}{\pi^{2}} \phi^{2} \frac{\mu F}{D^{2}} r \\
N_{Y}=\frac{n^{2} D L^{3} \mu F}{6\langle\hat{u}\rangle}\left(\left\langle p_{1}{ }^{3} p_{2}\right\rangle-\left\langle p_{1} p_{2}{ }^{3}\right\rangle\right)=\frac{4}{\pi^{2}} C \frac{b}{C} \phi^{3} \frac{\mu F}{D^{2}} \frac{r^{4}}{\ln ^{3 / 2} r}
\end{gathered}
$$

Note that the obtained equations (19) and (20) could be applied for any attractive interaction between fibres and are not restricted to a specific type or a specific expression for the resulting attractive force, like the one given in (4). Since little is known about electrokinetic properties of the CNT analyzed in the present paper and about the separation $h$ between the CNT surfaces, it is more convenient to use the colloidal force $F$, or rather a product $\mu \mathrm{F}$, as an adjustable parameter of the model.

We will proceed now to the comparison of the shear and normal stress predicted by our model with experiments of Natale et al. (2014) on the CNT suspensions. In addition, the predictions of the shear yield stress will be compared to the experiments of Rahatekar et al. (2009).

\section{Comparison with experiments}

\subsection{Flow curves}

In experiments, the CNT were dispersed in a Newtonian epoxy resin having a viscosity $\eta_{0}=12.3 \mathrm{~Pa} \cdot \mathrm{s}$ (Natale et al. (2014)). As stated in assumption (1) of $\S 2.1$, the average CNT diameter and average aspect ratio takes the values of $D \approx 16 \mathrm{~nm}$ and $r \approx 160$. The experimental and theoretical flow curves (shear stress versus shear rate dependencies) are shown in figure 2 for four different CNT volume fractions $\phi$ ranging from 0.59 to $2.36 \%$. First, we remark that the experimental flow curves (symbols in figure 2) have a rounded shape corresponding to a power law behaviour at low shear rates $\dot{\gamma}<10 \mathrm{~s}^{-1}$ followed by a linear segment at higher shear rates $\dot{\gamma}>10 \mathrm{~s}^{-1}$. Thus, the shear stress of CNT suspensions can be described by the Binhgam law, $\sigma_{12}=\sigma_{Y}+\eta_{s} \dot{\gamma}$ at $\dot{\gamma}>10 \mathrm{~s}^{-1}$ with the apparent shear yield stress 
$\sigma_{Y}$ obtained by a linear extrapolation of the flow curves to zero shear rate. This yield stress should not be confounded with the real yield stress which appears in some structured fluids and is considered to be the threshold stress required for the onset of the flow when the structure breaks or becomes slipping on the rheometer walls (Barnes (1999)). Qualitatively, our model (solid lines in figure 2) reproduces the linear segments of the flow curves but fails to capture the initial rounded segment. This is likely because we ignore particle aggregation. This phenomenon should seriously impact the suspension rheology at low shear rates. The aggregate size is expected to decrease with increasing shear rate resulting in a shear thinning manifested by the rounded shape of the flow curves. All the aggregates are expected to be broken at high shear rates. Individual particles continue to interact with each other at high shear rates and result in linear segments of the flow curves. Thus, our model can be applied safely only for high enough shear rates corresponding to the non-aggregated state.

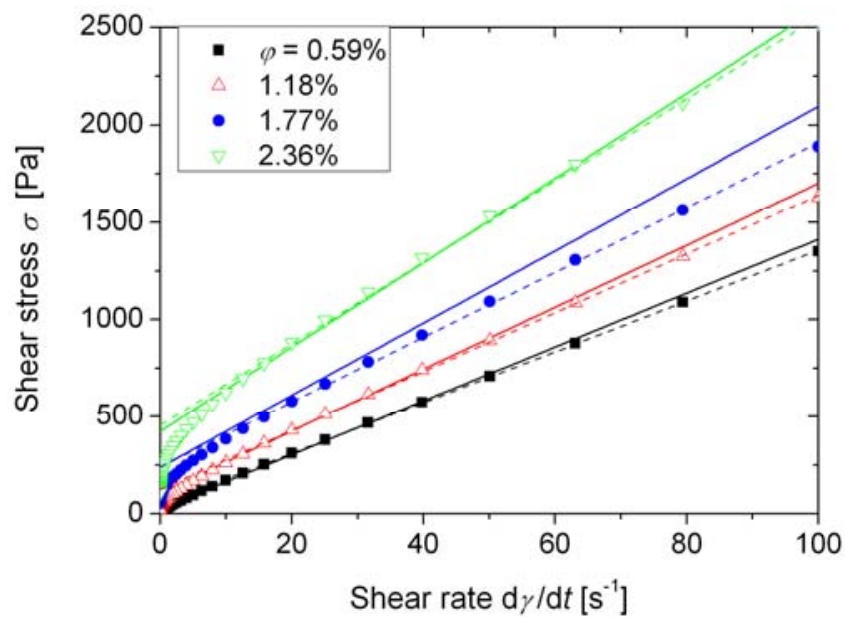

Figure 2. (Colour online) Experimental and theoretical flow curves of the CNT suspensions at different particle volume fractions $\phi$. The symbols correspond to experimental data of Natale et al. (2014), the dashed lines to the linear fit of the experimental data in the shear rate range $10 \leq \dot{\gamma} \leq 100 \mathrm{~s}^{-1}$, the solid lines correspond to the theory [equation (19.1) with $k=25$ and $\mu F=7.5 \times 10^{-13} \mathrm{~N}$ ]. The values of the suspension volume fraction $\phi$ are given in the figure legend.

Quantitatively, the model fits reasonably well the experimental data of Natale et al. (2014) at $\dot{\gamma}>10 \mathrm{~s}^{-1}$. According to equation (19.1), the two free parameters used for the fit of the flow curves are the product $\mu F$ of the friction coefficient by the colloidal force and the dimensionless constant $k$ intervening into equation (1) for the lubrication force. The best fit is obtained for $k=25$ and $\mu F=7.5 \times 10^{-13} \mathrm{~N}$.

\subsection{First normal stress difference}

Experimental and theoretical (data of Natale et al. (2014)) shear rate dependencies of the first normal stress difference for four different CNT volume fractions are plotted in figure 3. The experimental dependencies (symbols in figure 3) show the similar trend as the flow curves plotted in figure 2 . The normal stress difference seems to be insignificant at zero shear rate; a smooth power-law increase of the normal stress at low shear rates $\dot{\gamma}<30 \mathrm{~s}^{-1}$ is followed by a linear segment at higher shear rates $\dot{\gamma}>30 \mathrm{~s}^{-1}$. Thus, at $\dot{\gamma}>30 \mathrm{~s}^{-1}$ the 
experimental dependencies can be fitted by a linear trend $N_{1}=N_{Y}+\eta_{n} \dot{\gamma}$ similar to the Bingham law for the shear stress. The apparent normal yield stress $N_{Y}$ is found by a linear extrapolation of the experimental $N_{1}$ versus $\dot{\gamma}$ dependencies to zero shear rate. The relative yield contribution $N_{Y} / N_{1}$ to the normal stress appears to be more important than that $\sigma_{Y} / \sigma_{12}$ to the shear stress. This means that colloidal forces have more effects on the normal stress than on the shear stress. This could explain the difference between the shear rates $\dot{\gamma} \approx 30 \mathrm{~s}^{-1}$ and $\dot{\gamma} \approx 10 \mathrm{~s}^{-1}$ corresponding to the onset of the linear rheological behaviour of the normal and shear stresses, respectively.

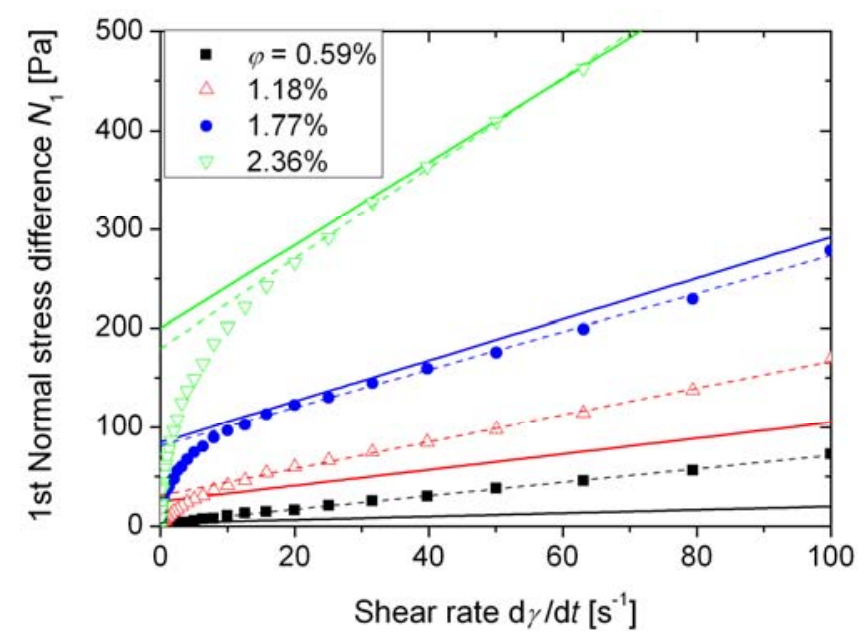

Figure 3. (Colour online) Experimental and theoretical shear rate dependencies of the first normal stress difference for the CNT suspensions at different particle volume fractions $\phi$. The symbols correspond to experimental data of Natale et al. (2014), the dashed lines to the linear fit of the experimental data in the shear rate range $30 \leq \dot{\gamma} \leq 100 \mathrm{~s}^{-1}$, the solid lines correspond to the theory (19.2). The suspension volume fraction $\phi$ is given in the figure legend.

The experimental curves were fitted by equation (19.2) of our model with the free parameters $k, \mu F$ and $C$. The fit was performed over the linear segment of the $N_{1}$ versus $\dot{\gamma}$ dependency since our model ignores particle aggregation that, as explained before, is likely responsible for the rounded shape of the experimental curves at low shear rate. The values of the first two parameters were taken to be the same as for the fitting of the flow curves: $k=25$ and $\mu F=7.5 \times 10^{-13} \mathrm{~N}$. Thus, the fit was performed using the remaining free parameter - the dimensionless constant $C$ appearing in equation (13) for the rotary diffusivity. The best fit corresponds to the value $C=3.7 \cdot 10^{-5}$. Strictly speaking the weak diffusion limit is hardly satisfied: at the highest volume fraction, we get $C \phi r_{e}^{3} \approx 0.6$ instead of $C \phi r_{e}^{3} \ll 1$ assumed in the model. However, numerical resolution of the Fokker-Planck equation shows that the approximation $C \phi r_{e}^{3} \ll 1$ still gives reasonable estimates of the moments $\langle\mathbf{p p p p}\rangle$ up to $C \phi r_{e}^{3} \sim 1$. For more quantitative comparison with experiments, we performed a linear fit of the experimental data in the shear rate range $30 \leq \dot{\gamma} \leq 100 \mathrm{~s}^{-1}$, individually for each particle concentration (cf. dashed lines in figure 3). As is seen from figure 3, the model seems to 
correctly predict the apparent normal yield stress $N_{Y}$ (ordinate at the origin) but gives poorer predictions for the slopes of $N_{1}$ versus $\dot{\gamma}$ dependencies.

\subsection{Apparent yield stress}

In order to better analyze the apparent yield stress predicted by our model, the concentration dependencies of both shear and normal yield stresses are plotted in figure 4 . The experimental points are obtained as the ordinates at the origin of the lines corresponding to individual linear fit of each experimental curve $\sigma(\dot{\gamma})$ or $N_{1}(\dot{\gamma})$ (dashed lines in figures 2 and 3). The theoretical concentration dependencies $\sigma_{Y}(\phi)$ and $N_{1}(\phi)$ are calculated using equation (21). Figure 4 shows a relatively good correspondence between experiments of Natale et al. (2004) and our model. Therefore, we may conclude that our model is able to capture the concentration effect on the apparent yield stress of the CNT suspension: according to equation (21), the shear yield stress is proportional to the concentration squared, $\sigma_{Y} \propto \phi^{2}$, while the normal yield stress - to the third power of the concentration, $N_{Y} \propto \phi^{3}$. From the theoretical point of view, $\sigma_{Y} \propto \phi^{2}$ behaviour comes from the pair-wise interactions between fibres, and $N_{Y} \propto \phi^{3}$ behaviour is the result of the combination of pair interactions (giving $\phi^{2}$ contribution to the stress) and rotational diffusivity linear in $\phi$ (cf. equation (13)).

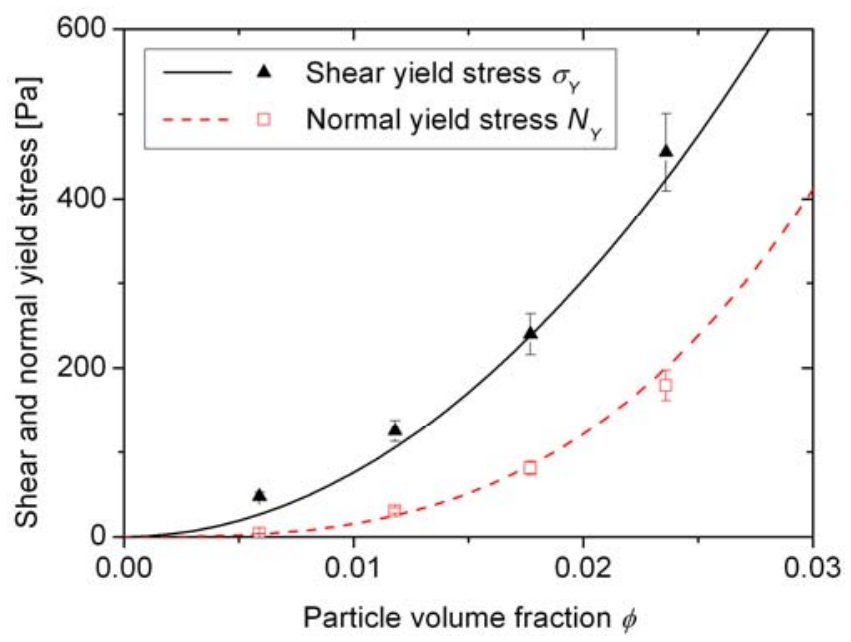

Figure 4. (Colour online) Experimental and theoretical dependencies of the shear and normal apparent yield stresses on particle volume fraction. Symbols correspond to the experiments of Natale et al. (2014) and solid lines to the theory

In experiments of Natale et al. (2014) the shear stress seems to be insignificant at zero shear rate (cf. figure2), so, the suspension does not exhibit any real shear yield stress. Thus, until now we analyzed the apparent yield stress obtained as an intercept of the linear extrapolation of the experimental flow curves onto zero shear rate. However, some other authors report the real yield behaviour of CNT/epoxy suspensions, corresponding to the onset of the flow (Rahatekar et al. (2009); Hobbie (2010)). This real yield stress likely appears as a result of stronger colloidal interactions than those between CNTs used in Natale's experiments. Both types of CNT have quite different diameter and aspect ratio $(D=16$ and 
$r=160$, in Natale's experiments and $D=50 \mathrm{~nm}$ and $r=1200$ and 80 in Rahatekar's experiments) and, as a consequence, quite different stress levels. Therefore, to compare the three sets of experimental data with our theory, it is convenient to introduce a dimensionless shear yield stress, $\sigma_{Y} D^{2} /(\mu F)$, normalized by a characteristic "interaction" stress $\mu F / D^{2}$. Our model (21.1) predicts that the dimensionless yields stress scales as $\sigma_{Y} D^{2} /(\mu F)=16 \phi^{2} r / \pi^{2} \propto \phi^{2} r$. To check whether this tendency holds for the three sets of experimental data, we plot in figure 5 the experimental and theoretical dependencies of the dimensionless yield stress on the factor $\phi^{2} r$.

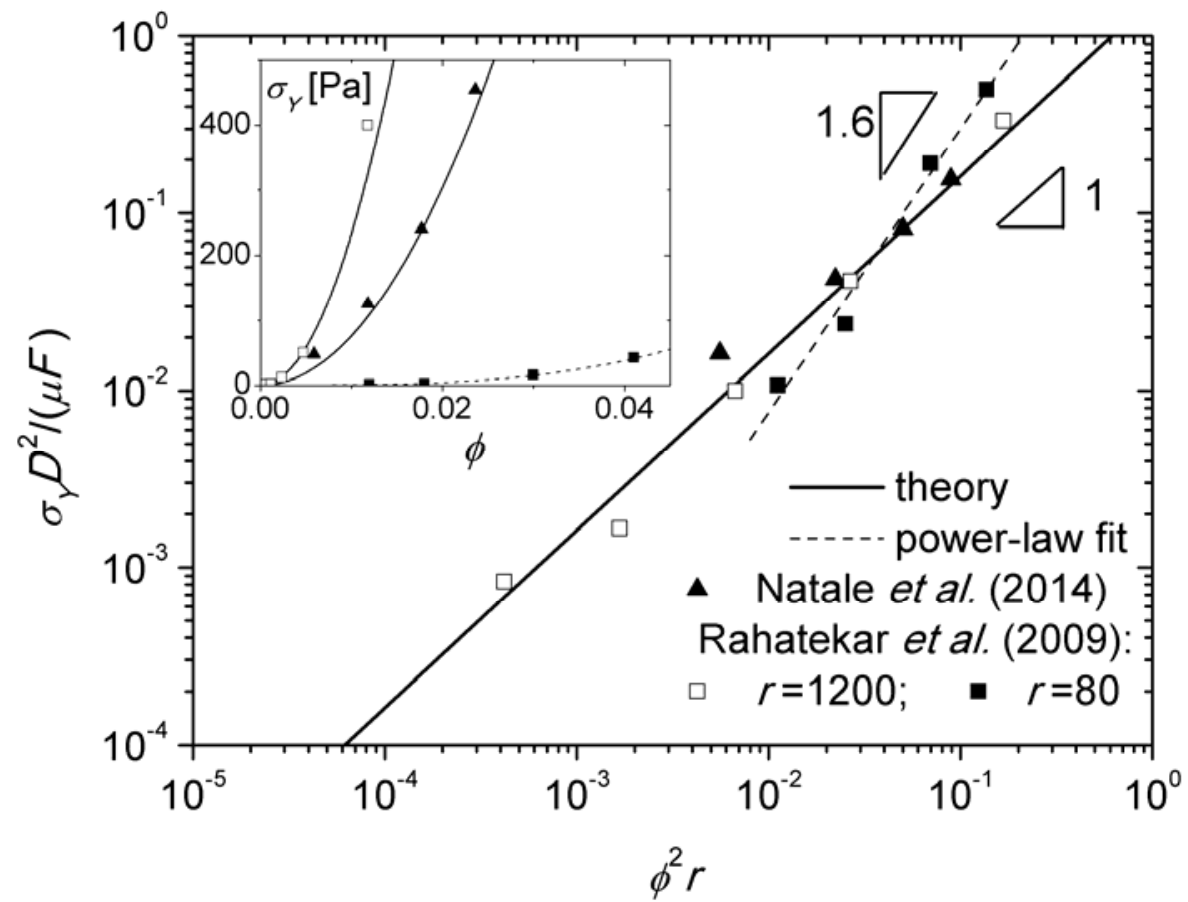

Figure 5. Dimensionless apparent yield stress as function of $\phi^{2} r$. The inset shows the dependency of the dimensional yield stress on particle concentration for the three sets of experimental data (symbols). The solid lines represent theoretical fits to the experimental data of Natale et al. (2014) and Rahatekar et al. (2009), while the dashed line is a power law fit to the data of Rahatekar et al. (2009) for CNT aspect ratio $r=80$.

The experimental values of the dimensionless yield stress have been obtained by fitting the experimental curves to the theoretical ones using the product $\mu F$ as single adjustable parameter. The best fit corresponds to the values $\mu F=3 \cdot 10^{-12} \mathrm{~N}$ and $2 \cdot 1 \cdot 10^{-13} \mathrm{~N}$ for $r=1200$ and 80 in Rahatekar's experiments, while $\mu F=7.5 \cdot 10^{-13} \mathrm{~N}$ for Natale's data with $r=160$ (the same value has been used above for the fit of the Natale's data shown in Figs. 2-4), The inset of Fig. 5 shows a concentration dependency of the dimensional yield stress. As inferred from this figure, the experimental data for the highest aspect ratios $r=160$ and 1200 respect the $\phi^{2} r$ behaviour predicted by our model for the dimensionless yield stress. The concentration dependency of the dimensional yield stress is also correctly predicted for these two data. For shorter particles, $r=80$, used in Rahatekar's experiments, a stronger concentration behaviour is observed. More precisely, the dimensional yield stress is best fitted by a power law trend with an exponent $3.2\left(\sigma_{Y} \propto \phi^{3.2}\right.$, dashed line in the inset of Fig. 5). This gives the exponent 1.6 of the dimensionless yield stress versus $\phi^{2} r$ (i.e. $\sigma_{Y} D^{2} /(\mu F) \propto\left(\phi^{2} r\right)^{1.6}$, dashed line in Fig.5). 
This discrepancy with our theory is likely connected to strong aggregation (ignored in the model) of the CNTs with $r=80$, while, at $r=1200$, the suspension is much less aggregated, as revealed by optical microscopy imaging reported by Rahatekar et al. (2009).

The value of the product $\mu F$ found by the fit of the theoretical model to experimental results allows estimation of the average separation $h$ between particle surfaces at the contact points. The electrostatic repulsion between untreated CNTs, used in Natale's and Rahatekar's experiments, is expected to be negligible and the value of $h$ is retrieved from (4.3) with a zero second term in the right hand side, namely: $h=(A \cdot D / 12 F)^{1 / 2}$. Thus, using a value $\mu=0.3$ for the friction coefficient [Petrich \& Koch (1998)] and $A \sim 10^{-20} \mathrm{~J}$ for the Hamaker constant [see Appendix B], we obtain the value $h \approx 2.3 \mathrm{~nm}$ for $r=160$ and $D=16 \mathrm{~nm}$ (Natale's data) and $h \approx 2.0 \mathrm{~nm}$ for $r=1200$ and $D=50 \mathrm{~nm}$ (Rahatekar's data). These values seem to be realistic and satisfy the limit of validity $h \ll D$ of assumptions (4) and (5) of $\S 2.1$.

It is also worth noticing that in the general case of 3D shear or extensional flows, our model predicts an anisotropic yield stress, as follows (see the last term of (18)):

$$
\begin{gathered}
\sigma^{Y}{ }_{i k}=\frac{8}{3 \pi^{2}} \phi^{2} \frac{\mu F}{D^{2}} r \frac{\left\langle p_{i} p_{k} p_{l} p_{m}\right\rangle}{\langle\hat{u}\rangle} \hat{\gamma}_{l m} \\
\langle\hat{u}\rangle=\frac{\langle|\mathbf{u}|\rangle}{L \dot{\gamma}}=\frac{1}{L^{3} \dot{\gamma}} \int_{\mathbf{p}^{\alpha}} \psi_{\alpha} d \mathbf{p}^{\alpha} \int_{\mathbf{p}^{\beta}} \psi_{\beta} d \mathbf{p}^{\beta} \int_{-L / 2}^{L / 2} \int_{-L / 2}^{L / 2}|\mathbf{u}| d s_{\alpha} d s_{\beta}
\end{gathered}
$$

where $\hat{\gamma}_{l m}=\gamma_{l m} / \dot{\gamma}$. In the particular case of simple shear flow in low diffusion limit, considered in the present paper, the equation (22.2) for $\langle\hat{u}\rangle$ reduces to equation (17.2). Our expression (22.1) for the yield stress tensor differs from the one derived recently by Férec et al. (2015):

$$
\begin{gathered}
\sigma^{Y}{ }_{i k}=\frac{8}{\pi^{2}} \phi^{2} k \frac{K}{D} r\left\langle\frac{p_{i} p_{k} p_{l} p_{m} \sin \delta}{\hat{u}}\right\rangle \hat{\gamma}_{l m} \\
\hat{u}=\left|p_{n} p_{s} \hat{\gamma}_{n s}\right|
\end{gathered}
$$

where $K$ is the consistency measured in $\mathrm{N} / \mathrm{m}, k$ is the dimensionless interaction parameter describing the intensity of inter-fibre forces and $\sin \delta=\left|\mathbf{p}^{\alpha} \times \mathbf{p}^{\beta}\right|$ (cf. figure 1). The last expression has been derived by setting to zero the value of the power-law index $\kappa$ in the phenomenological expression $\mathbf{F} \propto|\mathbf{u}|^{\kappa-1} \mathbf{u}$ for the interaction force between fibres. The physical meaning of the consistency $K$ has not been clarified. In contrast to the phenomenological approach adopted in this previous model, the present model introduces realistic physical interactions between fibres (colloidal, solid friction and lubrication forces) and predicts the yield stress tensor in terms of the friction force. Comparing equations (22.1) and (23) allows us to identify the scaling of the consistency in the model of Férec et al. (2015) in terms of measurable physical quantities: $K=O(\mu F / D)$. Another difference consists in the 
fact that the orientation tensor in (23) contains the Onsager potential $\sin \delta=\left|\mathbf{p}^{\alpha} \times \mathbf{p}^{\beta}\right|$ since, in the model of Férec et al. (2015), the interaction force is not supposed to be inversely proportional to $\left|\mathbf{p}^{\alpha} \times \mathbf{p}^{\beta}\right|$, like in the present theory (cf. discussion below equation (18)). Finally, the two models use different assumptions for the estimation of the absolute value $|\mathbf{u}|$ of the relative velocity of the fibres (cf. equations 17 and 22.2 for the present model and (23.2) for the model of Férec et al. (2015)). Notice that both models produce the same concentration behaviour of the yield stress, $\sigma_{Y} \propto \phi^{2}$, coming for pair-wise interactions.

In what concerns the aspect ratio effect, recall that the current model is developed for the high aspect ratio limit $r \gg 1$ predicting the shear yield stress proportional to $r$. The model is unable to provide quantitative predictions for lower aspect ratios, say $r<10$. However, qualitatively, the mean number of contact points per particle (coordination number) is much higher for long fibres than for spheres, at the same particle volume fraction. Therefore, at particle concentrations, as low as $\phi=0.02$, the effect of van der Waals forces is expected to be much lower for spherical particle suspensions than for long fibre suspensions.

\section{Concluding remarks}

This work is focused on the modelling of the shear and normal stresses in fibre suspensions that are subjected to a simple shear flow in the presence of short-range lubrication and overall attractive colloidal forces between fibres. The static friction force between fibres is also taken into account and supposed to be proportional to the attractive resultant colloidal force. The model is applied to the experimental data of Natale et al. (2014) and Rahatekar et al. (2009) on the suspensions of CNT dispersed in a Newtonian epoxy resin, however, it can also be used for other rod-like particle suspensions with attractive interactions.

The contact lubrication and solid friction forces are estimated in simple Cartesian reference frame and result in objective orientation and stress tensors. Following the approach of Djalili-Moghaddm \& Toll (1995) and Férec et al. (2009), these forces are averaged over the contact probability. The mean force exerted by neighbouring fibres to a test fibre appears to be zero and results in an affine translational motion. Furthermore, the deterministic angular motion of fibres is also unaffected by the short-range forces in agreement with DjaliliMoghaddm \& Toll (1995), as opposed to the work of Férec et al. (2009) who have used the co-rotational frame. These conclusions apply only under assumption of perfect sliding contacts in all contact points. The presence of sticky contacts should promote formation of fibre network (Switzer and Klingenberg (2004)). As a consequence, this could break the affinity of the translational motion and affect the rotational motion. Such an aggregation scenario is ignored in the present model.

The short-ranged interactions could in principle influence the rotary diffusivity of fibres and affect their stochastic rotational motion. However, in the weak diffusion $D_{r} r_{e}^{3} / \dot{\gamma} \ll 1$ and high aspect ratio $r \gg 1$ limits employed in the present work, the rotary 
diffusivity does not intervene into the component $\left\langle p_{1}^{2} p_{2}^{2}\right\rangle$ of the orientation tensor appearing in the shear stress but does into the goniometric factor $\left\langle p_{1}^{3} p_{2}\right\rangle-\left\langle p_{1} p_{2}{ }^{3}\right\rangle$ appearing in the first normal stress difference. The effect of the colloidal interactions on the rotary diffusivity was neglected. The combined effect of the long and short-ranged hydrodynamic interactions was taken into account in a phenomenological way, assuming $D_{r} \propto \phi \dot{\gamma}$. In this way, the factor $\left\langle p_{1}^{3} p_{2}\right\rangle-\left\langle p_{1} p_{2}^{3}\right\rangle$ appeared to be independent of shear rate and proportional to the particle concentration.

The particle stress was shown to be the sum of two contributions as follows: (a) the stress coming from hydrodynamic interaction between the fibres and the suspending liquid (with the rheological coefficients borrowed from the slender body theory in the dilute limit); (b) the stress coming from solid friction between fibres and proportional to the resultant attractive colloidal force. In the considered experimental conditions (Natale et al. (2014)), the lubrication force does not seem to provide any direct contribution to the particle stress but may influence the rotary diffusivity of the fibres. Our model predicts a simple Bingham law for both the shear stress and the first normal stress difference with the apparent shear and normal yield stresses proportional to $\phi^{2}$ and $\phi^{3}$, respectively. The $\sigma_{Y} \propto \phi^{2}$ trend is explained by pair colloidal interactions between fibres. The behaviour $N_{1} \propto \phi^{2}\left(\left\langle p_{1}^{3} p_{2}\right\rangle-\left\langle p_{1} p_{2}^{3}\right\rangle\right) \propto \phi^{3}$ stands for the synergy between the attractive colloidal forces (responsible for the $\phi^{2}$ contribution) and hydrodynamic interactions, implicitly included to the rotary diffusivity and giving $\left\langle p_{1}^{3} p_{2}\right\rangle-\left\langle p_{1} p_{2}{ }^{3}\right\rangle \propto \phi$. The second normal stress difference is negligible with respect to the first normal stress difference in the same way as in non-Brownian suspensions where fibres interact only through hydrodynamic forces (Larson (1999)).

The experiments on CNT suspensions also reveal apparent Bingham behaviour. The model captures well the quadratic concentration behaviour of the yield stress for long CNT particles used in Rahatekar et al. (2009) experiments $(r=1200)$ and Natale et al. (2014) experiments $(r=160)$, but fails to predict the $\phi^{3.2}$ trend for shorter CNTs, $r=80$, used by Rahatekar, likely because of strong aggregation observed for those particles. The flow curves in Natale's experiments have a rounded shape at low shear rates, while our model predicts a linear shape with a pronounced yield stress. Such a discrepancy is tentatively explained by aggregation at low shear rates ignored by the present model. Besides all the simplifications, the model fits reasonably well to experimental $\sigma_{12}$ versus $\dot{\gamma}$ and $N_{1}$ versus $\dot{\gamma}$ dependencies at high shear rates $\left(\dot{\gamma}>10 \mathrm{~s}^{-1}\right.$ for $\sigma_{12}$ and $\dot{\gamma}>30 \mathrm{~s}^{-1}$ for $\left.N_{1}\right)$. This is a positive argument for the validity of our non-aggregation approach. In future, we plan to construct a more realistic model taking into account both an imperfect sliding and particle aggregation. This future model should allow us to capture the rounded shape of the flow curves at low shear rates. It would combine the features of the present microscopic approach (allowing a rather rigorous determination of the contact forces) with macroscopic aggregation-orientation models proposed by Ma et al. (2008) and Abisset-Chavanne et al. (2015). 


\section{Acknowledgements}

The authors are grateful to Prof. P.J. Carreau, Prof. M.C. Heuzey and Dr. G. Natale of Polytechnique Montréal for providing the experimental data. They also would like to acknowledge Région Provence - Alpes Côte d'Azur which funded one of them. This work was also supported by the Program of the Ministry of Education of the Russian Federation (project No. 3.12.2014/K).

\section{Appendix A. Demonstration of equation (14) for the stress tensor}

The particle stress averaged over the contact probability but not yet averaged over the fibre orientation reads (Landau \& Lifshitz (1987), Pokrovskiy (1978)):

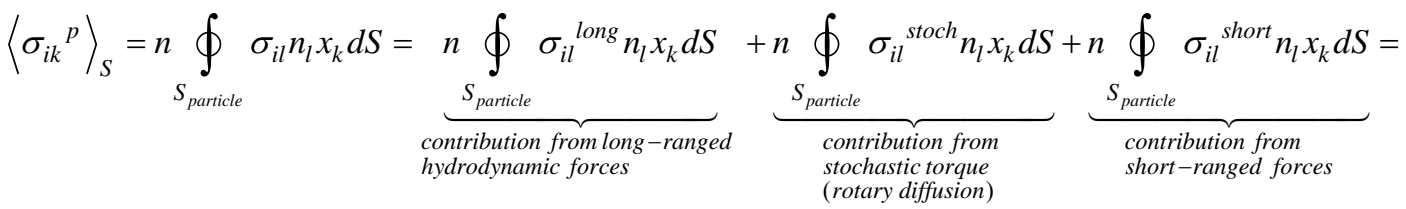

$$
\begin{aligned}
& =\underbrace{n V_{f} \eta_{0}\left\{4 \gamma_{i k}+\frac{\beta f^{\|}}{2}\left[\left\langle p_{i} p_{k} p_{l} p_{m}\right\rangle-\frac{1}{3} \delta_{i k}\left\langle p_{l} p_{m}\right\rangle\right] \gamma_{l m}\right\}-\frac{1}{2} n\left\{p_{i} p_{l} T_{l k}^{\text {hydro }}+p_{k} p_{l} T_{l i}^{\text {hydro }}\right\}+\frac{1}{2} n T_{i k}^{\text {hydro }}+} \\
& \text { contribution from long-ranged hydrodynamic forces } \\
& +\underbrace{n V_{f} \beta f^{\perp} \eta_{0} D_{r}\left[3\left\langle p_{i} p_{k}\right\rangle-\delta_{i k}\right]}_{\text {contribution from rotary diffusion }}+\underbrace{n p_{k} \underbrace{\int s_{\alpha} F_{i} d P}_{\varphi_{i}}}_{\begin{array}{c}
\text { contribution from } \\
\text { short-ranged forces }
\end{array}}= \\
& =\sigma_{i k}{ }^{p}{ }_{0}-\frac{1}{2} n\left\{p_{i} p_{l} T_{l k}^{\text {hydro }}+p_{k} p_{l} T_{l i}^{\text {hydro }}\right\}+\frac{1}{2} n T_{i k}^{\text {hydro }}+\frac{1}{2} n\left(\varphi_{i} p_{k}+\varphi_{k} p_{i}\right)+\frac{1}{2} n\left(\varphi_{i} p_{k}-\varphi_{k} p_{i}\right) \\
& \sigma_{i k}^{p}{ }_{0}=n V_{f} \eta_{0}\left\{4 \gamma_{i k}+\frac{\beta f^{\|}}{2}\left[p_{i} p_{k} p_{l} p_{m}-\frac{1}{3} \delta_{i k} p_{l} p_{m}\right] \gamma_{l m}\right\}+n V_{f} \beta f^{\perp} \eta_{0} D_{r}\left[3 p_{i} p_{k}-\delta_{i k}\right]
\end{aligned}
$$

where $T_{i k}^{\text {hydro }}$ is the "ik"-component of the tensor of the hydrodynamic torque acting on fibres, $\sigma_{i k}{ }_{0}^{p}$ is the stress generated by a test fibre at zero hydrodynamic torque in the absence of the short-ranged forces; $\varphi_{i}=\int s_{\alpha} F_{i} d P$; and other terms are explained below equation (14). The torque balance on a given fibre reads:

$$
T_{i k}^{\text {hydro }}+\left(\varphi_{i} p_{k}-\varphi_{k} p_{i}\right)=0
$$

Using this expression, we can show that

$$
\frac{1}{2} n\left\{p_{i} p_{l} T_{l k}^{\text {hydro }}+p_{k} p_{l} T_{l i}^{\text {hydro }}\right\}=\frac{1}{2} n\left(\varphi_{i} p_{k}+\varphi_{k} p_{i}\right)-n p_{i} p_{k} p_{l} \varphi_{l}
$$

Substituting (A3), (A4) into (A1) gives:

$$
\left\langle\sigma_{i k}^{p}\right\rangle_{S}=\sigma_{i k}^{p}{ }_{0}+n p_{i} p_{k} p_{l} \varphi_{l}
$$


The second term here stands for the longitudinal stress generated by short-ranged forces on the fibres and is given by (A2). Substituting (A2) into (A5), averaging over all possible fibre orientations and adding the solvent contribution we arrive at the final expression (14) for the stress tensor in the suspension.

\section{Appendix B. Estimation of the Hamaker constant}

The Hamaker constant for CNT/epoxy composites can be estimated using simplified Lifshitz theory applied to the case of non-retarded van der Waals interaction and completely screened electrostatic interaction [Russel et al. (1989)]:

$$
A \approx \frac{3 \hbar \omega_{U V}}{16 \sqrt{2}} \frac{\left(n_{f}^{2}-n_{s}^{2}\right)^{2}}{\left(n_{f}^{2}+n_{s}^{2}\right)^{3 / 2}}
$$

where $\hbar=1.055 \cdot 10^{-34} \mathrm{~J} \cdot \mathrm{s}$ is the Planck constant, $\omega_{U V} \approx 7.2 \cdot 10^{-15} \mathrm{rad} / \mathrm{s}-$ frequency of dominant relaxation of CNT in ultraviolet region, $n_{f} \approx 2.15$ and $n_{s} \approx 1.57$ - refractive indexes of CNT particles and epoxy matrix, respectively [Khalkhal et al. (2011)]. Estimations give a value $A \sim 10^{-20} \mathrm{~J}$.

\section{REFERENCES}

Abisset-Chavanne, E., Chinesta, F., Ferec, J., Ausias, G., \& Keunings, R. 2015 On the multiscale description of dilute suspensions of non-Brownian rigid clusters composed of rods. J. NonNewt Fluid. Mech. 222, 34-44

Abisset-Chavanne, E., Mezher, R., Le Corre, S., Ammar, A., \& Chinesta, F. 2013 Kinetic theory microstructure modelling in concentrated suspensions, Entropy, 15, 2805-2832.

Allaoui A., Bai S., Cheng H.M., \& Bai J.B. 2002 Mechanical and electrical properties of a MWNT/epoxy composite, Compos. Sci. Technol. 62, 1993-1998

Barnes H.A. 1999 The yield stress - a review or ' $\pi \alpha v \tau \alpha \rho \varepsilon \varepsilon^{\prime}$-everything flows? J. NonNewt. Fluid Mech. 81, 133-178

Batchelor, G. K. 1970 Slender-body theory for particles of arbitrary cross-section in Stokes flow. J. Fluid Mech, 44, 419-440.

Bennington, C. P. J., Kerekes R. J. \& Grace J. R. 1990 The yield stress of fibre suspensions. Canad. J. Chem. Eng., 68, 748-757.

Bergström, L. 1998 Shear thinning and shear thickening of concentrated ceramic suspensions. Colloids and Surfaces A 133, 151-155.

Brenner H. 1974 Rheology of a dilute suspension of axisymmetric Brownian particles, Int. J. Multiphase Flow 1, 195-341

Brenner S.L. \& Parsegian V.A. 1974 "A physical method for deriving the electrostatic interaction between rod-like polyions at all mutual angles", Biophys J. 14, 327-334

Chaouche, M., \& Koch, D. L. 2001 Rheology of non-Brownian rigid fibre suspensions with adhesive contacts. J. Rheol. 45, 369-382.

Christensen, R. M. 1991 Mechanics of Composite Materials. Krieger Publishing Company, Malabar.

Cui, H., \& Grace, J. R. 2007 Flow of pulp fibre suspension and slurries: A review. Int. J. 
Multiphase Flow, 33, 921-934.

Djalili-Moghaddam, M. \& Toll S. 2005 A model for short-range interactions in fibre suspensions. J. NonNewt. Fluid. Mech. 132, 73-83.

Doi, M., \& Edwards S. F. 1986 The Theory of Polymer Dynamics. Oxford University Press, New York.

Fan, Z. \& Advani, S. G. 2007 Rheology of multiwall carbon nanotube suspensions, J. Rheol. 51, 585-604.

Férec, J., Ausias, G., Heuzey, M. C., \& Carreau, P. J. 2009 Modelling fibre interactions in semiconcentrated fibre suspensions. J. Rheol. 53, 49-72.

Férec, J., \& Ausias G. 2015 Rheological modelling of non-dilute rod suspensions, in Rheology of non-spherical particle suspensions (ed. F. Chinesta \& G. Ausias) pp. 77-118. Elsevier, Oxford.

Férec J., Perrot A. \& Ausias G. 2015 Toward modelling anisotropic yield stress and consistency induced by fibre in fibre-reinforced viscoplastic fluids, J. NonNewt. Fluid Mech. 220, 69-76.

Folgar, F., \& Tucker C. L. 1984 Orientation behaviour of fibres in concentrated suspensions J.Reinf. Plast. Compos. 3, 98-119

Grossberg A. Yu., \& Khokhlov A.R. 1981 Statistical theory of polymeric lyotropic liquid crystals. Adv. Polymer. Sci. 41, 53-97

Hinch, E. J., \& Leal, L. G. 1972 The effect of Brownian motion on the rheological properties of a suspension of non-spherical particles, J. Fluid. Mech. 52, 683-712.

Hobbie E.K., 2010 Shear rheology of carbon nanotube suspensions, Rheol. Acta 49, 323-334

Jeffery, G. B. 1922 The motion of ellipsoidal particles immersed in a viscous fluid. Proc. Royal Soc. London A 102, 161-179.

Khalkhal, F., Carreau, P. J., \& Ausias, G. 2011 Effect of flow history on linear viscoelastic properties and the evolution of the structure of multiwalled carbon nanotube suspensions in an epoxy. J. Rheol. 55, 153-175.

Landau, L. D. \& Lifshitz, E. M., 1987 Course of Theoretical Physics, Vol. 6: Fluid Mechanics 2nd Edition, Pergamon Press.

Larson R. G., 1998 The Structure and Rheology of Complex Fluids. Oxford University Press.

Leal, L. G. \& Hinch, E. J. 1971 The effect of weak Brownian rotations on particles in shear flow. J. Fluid. Mech. 46, 685-703.

Ma, W. K. A., Chinesta, F., Ammar, A., \& Mackley, M. R. 2008 Rheological modelling of carbon nanotube aggregate suspensions, J. Rheol. 52, 1311-1330.

Michot, L. J., Baravian, C., Bihannic, I., Maddi, S., Moyne, C., Duval, J. F., Levitz P., \& Davidson, P. 2008 Sol- Gel and Isotropic/Nematic Transitions in Aqueous Suspensions of Natural Nontronite Clay. Influence of Particle Anisotropy. 2. Gel Structure and Mechanical Properties. Langmuir 25, 127-139.

Mongruel, A., \& Cloitre, M. 1999 Shear viscosity of suspensions of aligned non-Brownian fibres". Rheol. Acta 38, 451-457.

Mueller, S., Llewellin, E. W., \& Mader, H. M. 2010. The rheology of suspensions of solid particles. Proc. Royal Soc. A 466, 1201-1228.

Natale G., Heuzey M. C., Carreau P. J., Ausias G. \& Férec J. 2014 Rheological Modelling of Carbon Nanotube Suspensions with Rod-Rod Interactions. AIChE J. 60, 1476-1487 
Natale G., Reddy N. K., Ausias G., Férec J., Heuzey M. C. \& Carreau P. J. 2015 Rheo-optical response of carbon nanotube suspensions J. Rheol. 59, 499-524.

Petrich, M. P., \& Koch, D. L. 1998 Interactions between contacting fibres, Phys. Fluids 10, 2111-2113.

Petrie, C. J. 1999 The rheology of fibre suspensions. J. NonNewt. Fluid. Mech. 87, 369-402

Pokrovskiy V.N., 1978 Statistical mechanics of diluted suspensions, Nauka, Moscow (in Russian).

Rahatekar, S. S., Koziol, K. K. K., Butler, S. A., Elliott, J. A., Shaffer, M. S. P., Mackley, M. R., \& Windle, A. H. 2006 Optical microstructure and viscosity enhancement for an epoxy resin matrix containing multiwall carbon nanotubes. J. Rheol. 50, 599-610.

Rahatekar S.S., Koziol K.K., Kline S.R., Hobbie E.K., Gilman J.W. \& Windle A.H. 2009, Length-dependent mechanics of carbon-nanotube networks. Adv. Mater. 21, 874-878.

Russell, W. B., Saville D. A., \& Schowalter W. R. 1989 "Colloidal dispersions” (Cambridge University Press, Cambrige)

Saarinen, T., Haavisto, S, Sorvari, A., Salmela, J., \& Seppa la J. 2014 "The effect of wall depletion on the rheology of microfibrillated cellulose water suspensions by optical coherence tomography", Cellulose 21, 1261-1275.

Schmid C. F. \& Klingenberg, D. J. 2000 Mechanical flocculation in flowing fiber suspensions, Phys. Rev. Lett. 84, 290.

Servais, C., Månson, J. A. E., \& Toll, S. 1999 Fibre-fibre interaction in concentrated suspensions: Disperse fibres". J. Rheol. 43, 991-1004.

Solomon, M. J., \& Boger, D. V. 1998 The rheology of aqueous dispersions of spindle-type colloidal hematite rods, J. Rheol. 42, 929-949.

Song, Y. S., \& Youn, J. R. 2005 Influence of dispersion states of carbon nanotubes on physical properties of epoxy nanocomposites. Carbon 43, 1378-1385.

Switzer, L. H., \& Klingenberg, D. J. 2004 Flocculation in simulations of sheared fibre suspensions. Int. J. Multiphase Flow 30, 67-87.

Toll, S., \& Månson, J. A. E. 1994 Dynamics of a planar concentrated fibre suspension with non-hydrodynamic interaction, J. Rheol. 38, 985-997.

Van der Schoot, P., \& Odijk, T. 1992 Statistical theory and structure factor of a semidilute solution of rodlike macromolecules interacting by van der Waals forces. J. Chem. Phys., 97, 515-524.

Wierenga, A., Philipse, A. P., Lekkerkerker, H. N., \& Boger, D. V. 1998 Aqueous dispersions of colloidal boehmite: Structure, dynamics, and yield stress of rod gels. Langmuir 14,55-65

Wolf, B., White, D., Melrose, J. R., \& Frith, W. J. 2007 On the behaviour of gelled fibre suspensions in steady shear. Rheol. Acta 46, 531-537.

Yamane Y., Kaneda Y. \& Doi M. 1994 Numerical simulations of semi-dilute suspensions of rodlike particles in shear flow. J. NonNewt. Fluid. Mech. 54, 405-421 